\title{
SARCOMATOID CARCINOMA IN
} A BLADDER DIVERTICULUM A RARE CASE

Sir,

Sarcomatoid carcinoma are those, which exhibit a prominent spindle cell component. They are aggressive tumours with a poor prognosis and should be differentiated from true carcinosarcoma. ${ }^{[1]}$ So far, only two cases of sarcomatoid carcinoma in a bladder diverticulum have been reported.

This 56-year-old patient presented with total painless hematuria of 1 -month duration. Physical examination was negative. CT examination showed a large bladder diverticulum containing an irregular contrast enhancing mass (Figure 1). On cystoscopy, a large diverticulum with a nodulopapillary tumour with keratin material on surface was seen. Biopsy of the tumour reported a highgrade spindle cell neoplasm. The patient underwent bilateral pelvic Imphadenectomy, radical cystoprostatectomy with MAINZ II

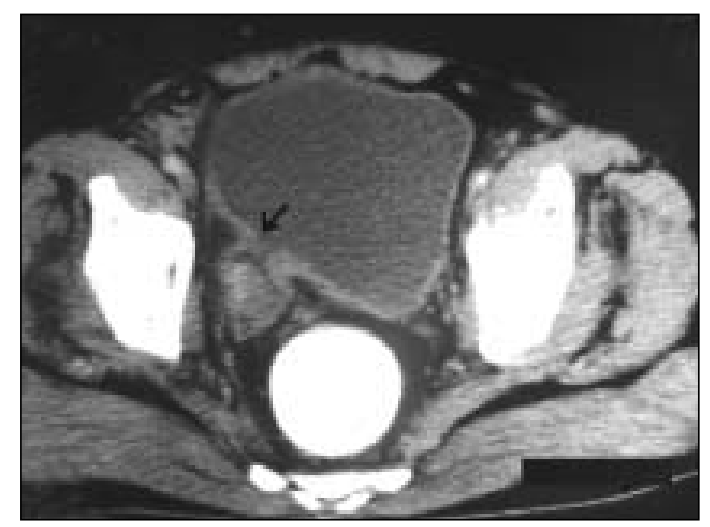

Figure 1: Contrast enhanced CT scan showing the neck of the diverticulum communicating with bladder 
ureterosigmoidostomy. The lymph nodes and resected margins were negative. Histopathology report proved to be sarcomatoid carcinoma. The tumor was composed of transitional cell carcinoma (TCC), sarcomatous spindle cells and the area of transition between TCC and spindle cells [Figure 2(inset)]. Immunohistochemical examination showed that the spindle cells and the area of transition were strongly positive for vimentin and weakly positive for cytokeratin (Figure 2). The histopathological and immunohistochemical transition between the TCC and the spindle cells implies that the sarcomatous elements originated from the TCC during tumour progression. The patient expired 6 months after surgery with extensive pelvic lymph node recurrence and lung metastasis.

The occurrence of a tumor inside a diverticulum is relatively rare. The TCC is the commonest tumor seen in a diverticulum. About 19 cases of intradiverticular sarcomas and carcinosarcomas have been reported in

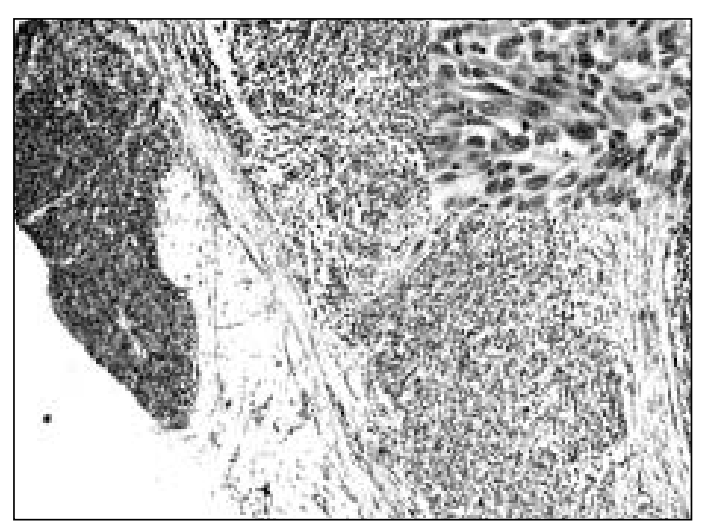

Figure 2: Microphotograph (100x) showing strongly positive vimentin areas. (Inset) Microphotograph (400 $x$ ) shows pleiomorphic sarcomatoid cells with hyper chromatic nuclei (H\&E staining) the literature. Of these only two cases are of sarcomatoid carcinoma. ${ }^{[2]}$ Patients with intradiverticular tumours usually present with hematuria, voiding difficulty, palpable mass. Neoplasms originating in a bladder diverticulum are characterized by early transmural invasion and tendency for higher histopathological grades. ${ }^{[3]}$ This makes early diagnosis and treatment imperative in these tumours. Filling defects caused by intradiverticular tumors may not always be visualized in intravenous urogram (IVU) and cystography. Cross-sectional imaging studies like US, CT, and magnetic resonance imaging (MRI) are being used with greater accuracy in the diagnosis and staging of intradiverticular tumours. MRI imaging in oblique planes can facilitate the diagnosis by demonstrating the neck of the diverticulum. Also, $T_{2}$ weighted images allow differentiation between tumor in a diverticulum and a necrotic extravesical mass. ${ }^{[4]}$ Cystoscopic findings of keratin materials and fast growing tumours also warrant special attention. As in our case, these signify a more sinister pathology than the usual TCC. Cystoscopic biopsy may not be diagnostic always as superficial biopsy may be inconclusive. The neoplasm's originating in a bladder diverticulum are characterized by early transmural invasion and high incidence of local recurrence. ${ }^{[5]}$ The previously reported cases treated with partial cystectomy had rapid intra abdominal recurrences and dismal prognosis. Our case demonstrates the accuracy of CT in detecting intradiverticular tumours. Obtaining a deep biopsy by cystoscopy is essential for diagnosis. The intradiverticular sarcoma and sarcomatoid carcinoma portend a poor prognosis even with aggressive surgical resection.

\section{REFERENCES}

1. Young RH, Wick MR, Mills SE. Sarcomatoid carcinoma of the urinary bladder. A clinicopathologic analysis of 12 cases and review of the literature. Am J Clin Pathol. 1988; 90:653-61

2 Kim MY, Jeon YS, Suh CH, Park WH. Sarcomatoid carcinoma arising from the diverticulum of the urinary bladder dome: a difficult diagnosis with imaging. Am. J. Roentgenol. 1999; 172: 1454-5

3. Yagci C, Atasoy C, Fitoz S, Akyar S. Cross sectional imaging findings in intradiverticular bladder tumora case report. Tani Girisim Radyol.2003; 9:452-5
4. Durfee SM, Schwartz LH, Panicek DM, Russo P. MR imaging of carcinoma within bladder diverticulum. Clinical Imaging 1997; 21:290-2

5. Cheng CW, Ng MT, Cheung HY, Sun WH, Chan LW, Wong WS, Lai FM. Carcinosarcoma of the bladder diverticulum and a review of the literature. Int J Urol. 2004; 11:1136-8.

T. A. KISHORE, S. BHAT, A. S. ALBERT, J. AUGUSTINE Kottayam, Kerala, India

Correspondence TA Kishore Department of Urology, Medical College, Kottayam - 686008 , Kertala, India, E-mail: kishoreta@yahoo.com Department of Urology and Pathology, Medical College,

Indian Journal of Medical Sciences is pleased to announce the launch of its website. The URL of the website is http://www.indianjmedsci.org.

The features of the site are:

- Free full text availability of articles in HTML as well as PDF

- Link to abstracts and full text from the cited references

- Link to PubMed abstracts of published articles by authors

- Link to related articles in PubMed

- Link from text of articles to various databases and search engines

- Facility to submit comments on articles

- Email notifications on new issue release

- Statistics of articles download and visits

- Structure based on OpenURL, DC Metadata and other international standards 